\title{
AN IMPROVED XFEM FOR THE POISSON EQUATION WITH DISCONTINUOUS COEFFICIENTS
}

\begin{abstract}
Discontinuous coefficients in the Poisson equation lead to the weak discontinuity in the solution, e.g. the gradient in the field quantity exhibits a rapid change across an interface. In the real world, discontinuities are frequently found (cracks, material interfaces, voids, phase-change phenomena) and their mathematical model can be represented by Poisson type equation. In this study, the extended finite element method (XFEM) is used to solve the formulated discontinuous problem. The XFEM solution introduce the discontinuity through nodal enrichment function, and controls it by additional degrees of freedom. This allows one to make the finite element mesh independent of discontinuity location. The quality of the solution depends mainly on the assumed enrichment basis functions. In the paper, a new set of enrichments are proposed in the solution of the Poisson equation with discontinuous coefficients. The global and local error estimates are used in order to assess the quality of the solution. The stability of the solution is investigated using the condition number of the stiffness matrix. The solutions obtained with standard and new enrichment functions are compared and discussed.
\end{abstract}

\section{Introduction}

Discontinuous problems can appear in different areas of engineering and solving them is still a challenging task for engineers and material sciences community. A challenging field where an accurate description of discontinuity is of vital importance is the modelling of failure in machine design. The failure may be a result of crack propagation in material, what is an example of a problem with strong discontinuity, e.g., the problem where the field quantity exhibits a rapid change across an interface. The problem where a weakdiscontinuity is observed is the

\footnotetext{
${ }^{1}$ Kielce University of Technology, Faculty of Management and Computer Modelling, Al. Tysiąclecia Państwa Polskiego 7, 25-314 Kielce, Poland.Email: stapor@tu.kielce.pl
} 
phase-change phenomena. The phase-change problem plays an important role in a number of industrial and natural processes. In this case, the discontinuity is present in the gradient of temperature. Another examples where proper modelling of weak discontinuity is important is the occurrence of the material interfaces, inclusions or holes.

The problem can be solved approximately using a wide range of numerical techniques. The overview of the meshfree methods can be found in [1] and [2]. In [3] authors present the implementation of the extended isogeometric analysis (IGA) which incorporates enrichment functions through the partition of unity method (PUM). In this paper, the solution of the Poisson equation with discontinuous terms using extended finite element method (XFEM) is considered. The XFEM was first used by Belytschko \& Black [4] to study crack propagation in materials. Merle \& Dolbow [5] and Chessa et al. [6] apply the XFEM to solve phase-change problems. The analysis of the one-dimensional physically non-linear phase-change problem is considered in [7].

The XFEM solution introduce the discontinuity through nodal enrichment function, and controls it by additional degrees of freedom. This allows one to make the finite element mesh independent of discontinuity location. The quality of the solution depends mainly on the assumed enrichment basis functions. However, the additional enrichment results in higher condition number of the stiffness matrix in comparison to the standard finite element approximation. To remedy the resulting poor conditioning, various approaches have been suggested in the literature, the review can be found in [8]. In the context of weak discontinuities, Hansbo et al. [9] and Wadbro et al. [10] improve the conditioning of the system by adding additional volume terms to the weak formulation of the problem. This results in increase of the solution error around the discontinuity. In [11] and [12] the authors developed the SGFEM (Stable Generalised Finite Element Method), for which the condition number is bounded and independent of the position of the interface. In general, the ill-conditioned system matrix of the XFEM solution is still a challenging issue.

In the paper, the new enrichment functions are proposed. The global and local error estimates are used in order to assess the solution error. The stability of the solution is investigated using the condition number of the stiffness matrix. The solution obtained with standard and the new enrichment functions are compared and discussed.

The outline of the paper is as follows. In Section 2, the problem considered throughout the paper is defined for one-dimensional space. In Section 3, the main futures of the XFEM are briefly recalled and a new sets of enrichment functions for one-dimensional case are defined. The solutions for one-dimensional example problem is presented and discussed in Section 4. The problem is extended to two-dimensional space in Section 5. Finally, the numerical solutions for twodimensional example problems are presented in Section 6 and 7. The solution error, condition number of a system and the convergence rates are analysed. 


\section{One-dimensional problem formulation}

Let us consider a one-dimensional domain $\Omega$ partitioned into subdomains, $\Omega_{L}$ and $\Omega_{R}$, respectively (Fig. 1). The subdomains are separated from each other by the interface $\Gamma_{I}$ at the point $\xi$. The Dirichlet condition is prescribed at the point $\Gamma_{D}$, while the Neumann condition is defined at $\Gamma_{N}$. The problem is described by Poisson ODE equation in the form

$$
\frac{\mathrm{d}}{\mathrm{d} x}\left(k(x) \frac{\mathrm{d}}{\mathrm{d} x} u(x)\right)+f(x)=0 \quad \text { in } \Omega
$$

with boundary conditions

$$
\begin{gathered}
u(x)=u_{D} \quad \text { at } \quad \Gamma_{D} \\
k \frac{\mathrm{d}}{\mathrm{d} x} u(x)=q_{N} \quad \text { at } \quad \Gamma_{N} .
\end{gathered}
$$

It is assumed that the coefficient $k(x)$ and function $f(x)$ are explicitly discontinuous across the interface boundary $\Gamma_{I}$, which can be written as follows

$$
\begin{aligned}
& k(x)=\left\{\begin{array}{lll}
k_{L}(x) & \text { in } & \Omega_{L} \\
k_{R}(x) & \text { in } & \Omega_{R}
\end{array}\right. \\
& f(x)=\left\{\begin{array}{lll}
f_{L}(x) & \text { in } & \Omega_{L} \\
f_{R}(x) & \text { in } & \Omega_{R} .
\end{array}\right.
\end{aligned}
$$

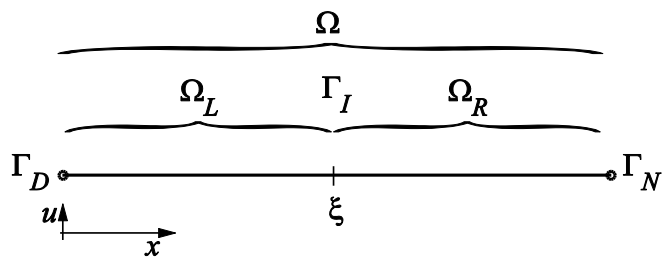

Fig. 1. One-dimensional domain $\Omega$ split into $\Omega_{L}$ and $\Omega_{R}$ by the interface $\Gamma_{I}$

The weak form of the problem is derived by multiplying the equation (1) by a test function $v(x)$ and integrating separately in each domain. As a result, we obtain the following weak form: find $u \in S$ such that

$$
\left(k(x) \frac{\mathrm{d}}{\mathrm{d} x} u(x), \frac{\mathrm{d}}{\mathrm{d} x} v(x)\right)=(f(x), v(x))-\left(v(x), q_{N}\right)_{\Gamma_{N}} \quad \forall v \in V
$$

with $S=\left\{u \backslash u \in H^{1}(\Omega),\left.u\right|_{\Gamma_{D}}=u_{D}\right\}$ and $V=\left\{v \backslash v \in H^{1}(\Omega),\left.v\right|_{\Gamma_{D}}=0\right\}$, where $S$ is the space of the trial solution, $V$ is the space of the test function, and $H^{1}$ is the first order Sobolev space. In the above expression, $(\cdot, \cdot)$ denotes the $L_{2}$ inner product over the domain $\Omega$. 


\section{Extended finite element method}

The finite element formulation of (6) is: find $u_{h} \in S_{h}$ such that

$$
\left(k(x) \frac{\mathrm{d}}{\mathrm{d} x} u_{h}, \frac{\mathrm{d}}{\mathrm{d} x} v_{h}\right)=\left(f, v_{h}\right)-\left(v_{h}, q_{N}\right)_{\Gamma_{N}} \quad \forall v \in V
$$

where $S_{h}$ and $V_{h}$ are finite element subspaces of $S$ and $V$, respectively. After the basic functions are introduced in $S_{h}$ and $V_{h}$, equation (7) can be written in the matrix form

$$
\mathbf{K u}=\mathbf{f}-\mathbf{q}
$$

where $\mathbf{u}$ is a vector of nodal unknowns, and $\mathbf{K}$ and $\mathbf{f}$ are the stiffness matrix and load vector, respectively. Their global counterparts are computed through the usual assembly procedure.

In the XFEM, the special functions reflecting the discontinuous field are introduced locally in the finite element approximation, which makes it possible to describe weak and strong discontinuities independent of the finite element mesh. The enriched area is limited only to elements cut by discontinuity interface, which does not increase the size of the problem significantly.

A solution characteristic of the problem is introduced by adding the enrichment term $u_{h}(x, \xi)_{E}$ to the standard finite element approximation $u_{h}(x)_{C}$

$$
u_{h}(x, \xi)=u_{h}(x)_{C}=u_{h}(x, \xi)_{E}
$$

where

$$
u_{h}(x)_{C}=\sum_{j \in I} N_{j}(x) u_{j}
$$

and $u$ is arbitrary quantity. The enrichment term $u_{h}(x, \xi)_{E}$ combines the enrichment functions $\Psi^{\alpha}(x, \xi)$ with a partition of unity (PU) functions $N_{j}(x)$ (usually element shape functions)

$$
u_{h}(x, \xi)_{E}=\sum_{j \in I} \sum_{\alpha=1}^{m} N_{j}(x) \Psi^{\alpha}(x, \xi) a_{j}^{\alpha}
$$

where $J$ is the set of nodes enriched by $\Psi^{\alpha}(x, \xi), a_{j}^{\alpha}$ are the additional degrees of freedom, $I$ is the set of all nodes, and $m$ is the number of enrichment functions and $\xi$ denotes the discontinuity location in the element.

It should be pointed out that the standard XFEM approximation does not satisfy the Kronecker property. This condition can be achieved by applying shifted-basis approximation [13], [14] to modify the enrichment function $\Psi^{\alpha}(x, \xi)$

$$
\Psi_{j}^{\alpha}(x, \xi)=\Psi^{\alpha}(x, \xi)-\Psi^{\alpha}\left(x_{j}, \xi\right)
$$

which leads to the desired property $u_{h}\left(x_{j}\right)_{E}=0$ and $u_{h}\left(x_{j}\right)=u_{j}$. 
The finite element mesh comprises standard elements, elements cut by the interface (reproducing elements), and elements that contain both the enriched and non-enriched nodes (blending elements). The approximation in the blending elements does not form a partition of unity; as a result, the convergence rate and accuracy of the method are reduced. In order to improve accuracy of the method, Tarancòn [15] suggests a fixed enrichment area, also known as geometrical enrichment. Fries [16] uses a ramp function over the transition layer so that the partition of unity property can be satisfied everywhere.

In this study, two approaches are considered. In the first, the standard absenrichment function with shifted-basis approximation is utilized

$$
\Psi_{j}(x, \xi)=|x-\xi|-\left|x_{j}-\xi\right| .
$$

In this approach, the enriched part of approximation in blending elements is not active, [17]. The equivalent approximation can be achieved using the function proposed by Moës et al. [18] or Babuska \& Banerjee [11]. In both cases, the enriched part of approximation is represented by a bilinear function which vanishes in blending elements. Example functions are presented in Fig. 2.

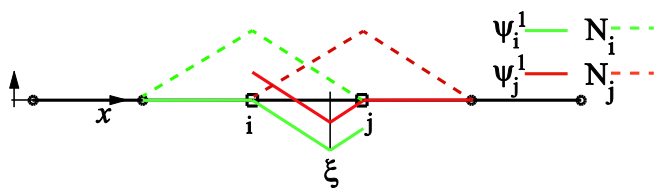

Fig. 2. Shifted-basis enrichment functions $\Psi^{1}(x, \xi)$ and PU function $N(x)$

In the second case, a new enrichment functions are proposed. The standard approximation (13) is extended to the fallowing

$$
\begin{aligned}
& \Psi_{j}^{1}(x, \xi)=\Psi_{j}(x, \xi) \\
& \Psi_{j}^{2}(x, \xi)=|x-\xi| g\left(x, x_{j}\right)-\left|x_{j}-\xi\right| .
\end{aligned}
$$

Four cases of the function $g\left(x, x_{j}\right)$ are considered, namely:

$$
\begin{aligned}
& \text { Case 1: } g\left(x, x_{j}\right)= \begin{cases}N_{1}\left(x_{j}\right) & \text { if } x<\xi \\
N_{2}\left(x_{j}\right) & \text { if } x>\xi\end{cases} \\
& \text { Case 2: } g\left(x, x_{j}\right)= \begin{cases}N_{1}(x) & \text { if } x<\xi \\
N_{2}\left(x_{j}\right) & \text { if } x>\xi\end{cases} \\
& \text { Case 3: } g\left(x, x_{j}\right)= \begin{cases}N_{1}(x) & \text { if } x<\xi \\
N_{2}(x) & \text { if } x>\xi\end{cases}
\end{aligned}
$$


Case 4: $g\left(x, x_{j}\right)= \begin{cases}N_{1}\left(x_{j}\right) & \text { if } x<\xi \\ N_{2}(x) & \text { if } x>\xi\end{cases}$

In Fig. 3, the functions for all cases are presented graphically. Thus, the enrichment term is defined by two functions $\Psi_{j}^{1}(x, \xi)$ and $\Psi_{j}^{2}(x, \xi)$.

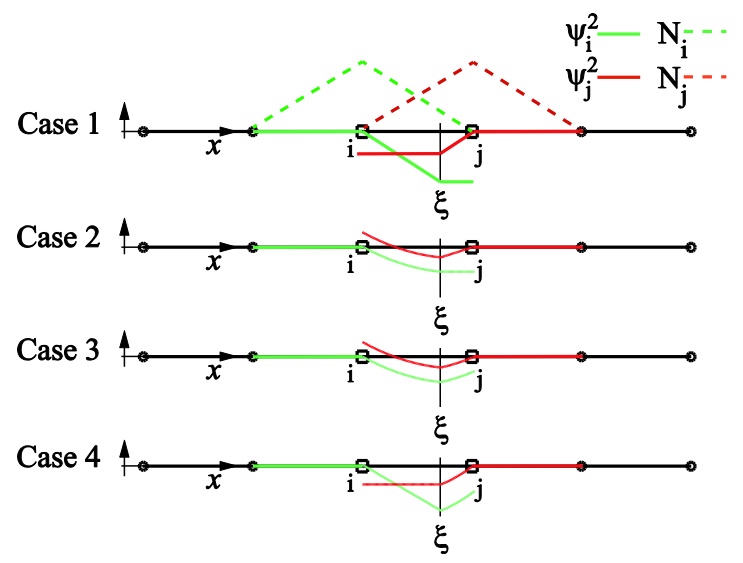

Fig. 3. Proposed enrichment functions $\Psi^{2}(x, \xi)$ for the cases $1,2,3$ and 4

The two-node finite element is used with standard $C^{0}$ continuity shape functions of the form

$$
\mathbf{N}(x)=\left[1-\frac{x}{l} \quad \frac{x}{l}\right]
$$

where $l$ is the length of the element and $x^{e} \in[0, l]$ is the local coordinate.

The element's degrees of freedom for the standard approach $\mathbf{u}_{S}$ and the new approach $\mathbf{u}_{N}$ are defined, respectively

$$
\begin{gathered}
\mathbf{u}_{S}=\left\{\begin{array}{llll}
u_{1} & u_{2} & a_{1} & a_{2}
\end{array}\right\} \\
\mathbf{u}_{N}=\left\{\begin{array}{llllll}
u_{1} & u_{2} & a_{1}^{1} & a_{2}^{1} & a_{1}^{2} & a_{2}^{2}
\end{array}\right\} .
\end{gathered}
$$

It should be noted that, when $\mathbf{K}$ and $\mathbf{f}$ are calculated, the integrands are discontinuous at the point $\xi$. In order to integrate properly these matrices and vectors, the domain of integration is partitioned into subdomains separated by the interface. The partitioning of enriched elements is done solely for the purpose of numerical integration. The element contribution to $\mathbf{K}$ and $\mathbf{f}$ are as follows

$$
\mathbf{K}=\int_{0}^{l} k \mathbf{B}_{X}^{T} \mathbf{B}_{X} \mathrm{~d} x
$$




$$
\mathbf{f}=\int_{0}^{l} \mathbf{N}_{X}^{T} f \mathrm{~d} x
$$

where

$$
\begin{gathered}
\mathbf{N}_{X}=\left[\begin{array}{ll}
\mathbf{N} & \mathbf{N} \boldsymbol{\Psi}
\end{array}\right] \\
\mathbf{B}_{X}=\frac{\mathrm{d}}{\mathrm{d} x} \mathbf{N}_{X}
\end{gathered}
$$

and

$$
\begin{aligned}
& \mathbf{N}_{X}=\left\{\begin{array}{l}
\mathbf{N}_{S}=\left[\begin{array}{lllll}
N_{1} & N_{2} & N_{1} \Psi_{1}^{1} & N_{2} \Psi_{2}^{1}
\end{array}\right] \\
\mathbf{N}_{N}=\left[\begin{array}{llllll}
N_{1} & N_{2} & N_{1} \Psi_{1}^{1} & N_{2} \Psi_{2}^{1} & N_{1} \Psi_{1}^{2} & N_{2} \Psi_{2}^{2}
\end{array}\right]
\end{array}\right. \\
& \mathbf{B}_{X}=\left\{\begin{array}{l}
\mathbf{B}_{S}=\frac{\mathrm{d}}{\mathrm{d} x} \mathbf{N}_{S} \\
\mathbf{B}_{N}=\frac{\mathrm{d}}{\mathrm{d} x} \mathrm{~N}_{N}
\end{array}\right.
\end{aligned}
$$

The subscripts $S$ and $N$ denote the standard and the new approximations, respectively. In the one-dimensional case, the location of discontinuity is represented by a single point, thus the explicit treatment does not cause any difficulties.

\section{One-dimensional numerical example}

Let us analyse the equation (1) in $\Omega=\langle 0,1\rangle$ with the following discontinuous terms:

$$
\begin{gathered}
k(x)= \begin{cases}k_{L}=2 & \text { in } \Omega_{L} \\
k_{R}=1 & \text { in } \Omega_{R}\end{cases} \\
f(x)= \begin{cases}f_{L}(x)=100 x^{2} & \text { in } \Omega_{L} \\
f_{R}(x)=0 & \text { in } \Omega_{R}\end{cases}
\end{gathered}
$$

where subdomains $\Omega_{L}$ and $\Omega_{R}$ are separated at the point $\xi=0.5$. The boundary conditions are defined as follows

$$
\begin{gathered}
u(x=0)=0 \\
k \frac{\mathrm{d}}{\mathrm{d} x} u(x=1)=-1 .
\end{gathered}
$$

The solutions are obtained for the meshes which deliberately do not align with discontinuity. For the first considered mesh, the domain is divided into five equallength finite elements. Discontinuity goes across element 3, so nodes 3 and 4 are enriched. The enriched nodes are marked with square, Fig. 4. 


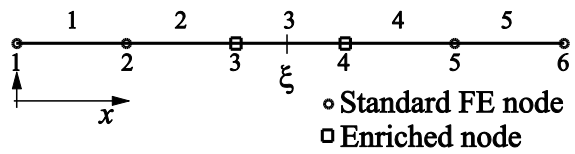

Fig. 4. An example mesh with enriched nodes

First, the problem is solved using standard enrichment functions (13). The solution is presented in Fig. 5. From the actual solution, there should not be any jump in $k \frac{\mathrm{d}}{\mathrm{d} x} u(x)$ at the interface point. This quantity can thus be treated as a local measure of analysis error. The proper estimation of the jump at the interface is of crucial interest in several physical problems, e.g., phase-change phenomena, where the movement of the interface depends on the jump in the heat flux at the interface. The quantity is calculated according to the formula

$$
\bar{q}=\left.k_{L} \frac{\mathrm{d}}{\mathrm{d} x} u(x)\right|_{\Gamma_{L}}-\left.k_{R} \frac{\mathrm{d}}{\mathrm{d} x} u(x)\right|_{\Gamma_{R}} .
$$

The analysis with standard enrichment gives $\bar{q}=0.76$ for five equal-length elements. As can be seen from Fig. 5, the approximation of the derivative is not
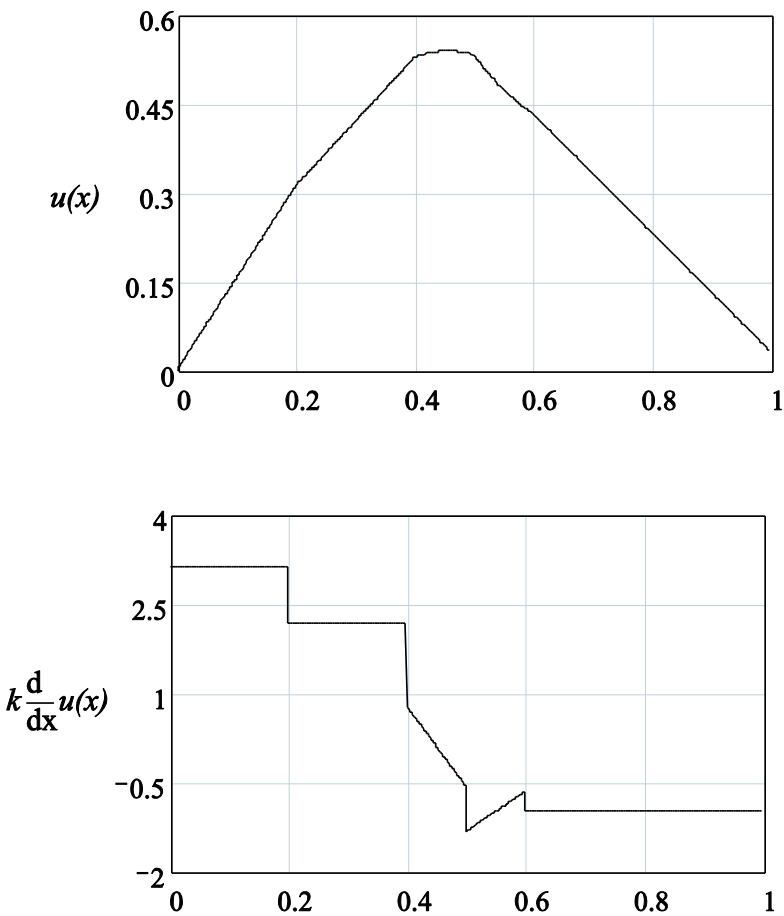

Fig. 5. Standard X-FEM solution $u(x)$ and its derivative $k \frac{\mathrm{d}}{\mathrm{d} x} u(x)$ 
optimal in the enriched element. This example demonstrates the inability of the standard XFEM approximation to accurately predict the solution in enriched element. It is due to the fact, that the higher order terms appear in the smooth part of approximation and cannot be compensated by regular FE shape functions. The similar problem occurs if original (non-shifted) approximation is applied, except that the spurious terms appears also in blending elements, [17].

The calculations are repeated for the enrichment functions (14). The results are presented in Fig. 6. The jump in derivative in this case is reduced to $\bar{q}=0.097$. The additional enrichment functions makes it possible to mitigate spurious deviation of the derivative in the element containing the interface. However, for the case 1 of the approximation, the stiffness matrix is severely ill-conditioned $\left(\sim 10^{15}\right)$. The results obtained for other cases are presented in Table 1. As can be seen, the enrichment functions for the case 2 mimic the local behaviour of the unknown solution of the underlying variational problem with minimal error, and the condition number is reduced over ten orders of magnitude in comparison to the case 1 . The calculated condition number is based on $L_{2}$ norm.

We now solve the problem again, using several different mesh spacing $h$, in order to assess the convergences of the method. In Fig. 7 and 8 the jump at the interface and the condition number are presented for all cases, respectively. The
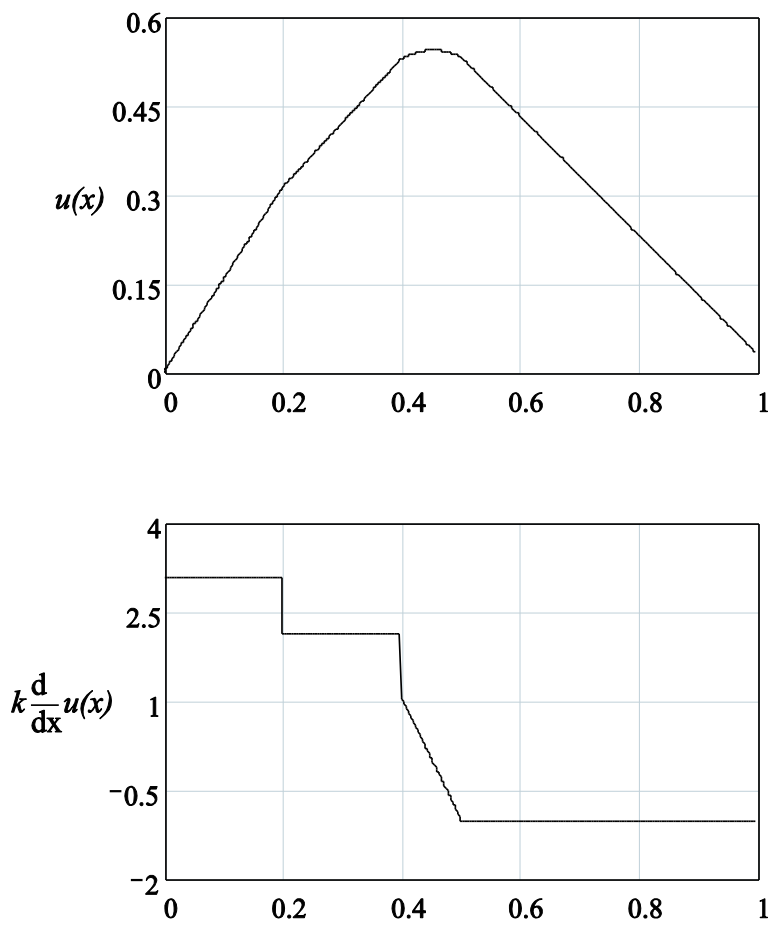

Fig. 6. Modified XFEM solution $u(x)$ and its derivative $k \frac{\mathrm{d}}{\mathrm{d} x} u(x)$ 
Solution error and condition number for different approximations tested

\begin{tabular}{|c|c|c|}
\hline $\begin{array}{c}\text { XFEM } \\
\text { Approximation }\end{array}$ & Error $\bar{q}$ & $\begin{array}{c}\text { Condition } \\
\text { number }\end{array}$ \\
\hline Standard & 0.76 & 624.079 \\
\hline Case 1 & 0.097 & $4.886 \cdot 10^{15}$ \\
\hline Case 2 & 0.027 & $7.425 \cdot 10^{4}$ \\
\hline Case 3 & 0.074 & $4.309 \cdot 10^{4}$ \\
\hline Case 4 & 0.097 & $1.472 \cdot 10^{5}$ \\
\hline
\end{tabular}

logarithmic scales are used for axes. As can be seen, the standard approximation allows for the least condition number of the stiffness matrix, however, the calculated error excides considerably the error for other cases. The influence of the position

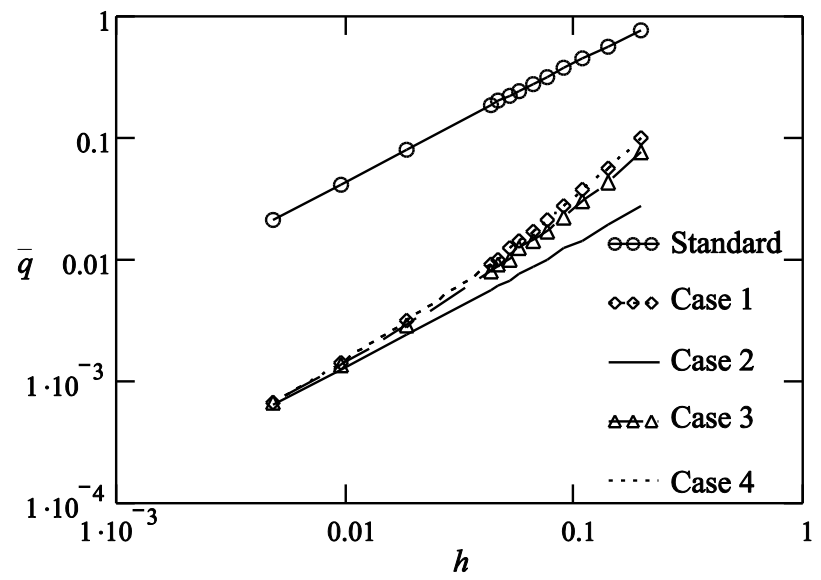

Fig. 7. Convergence rates for the calculated jump $\bar{q}$ at the interface

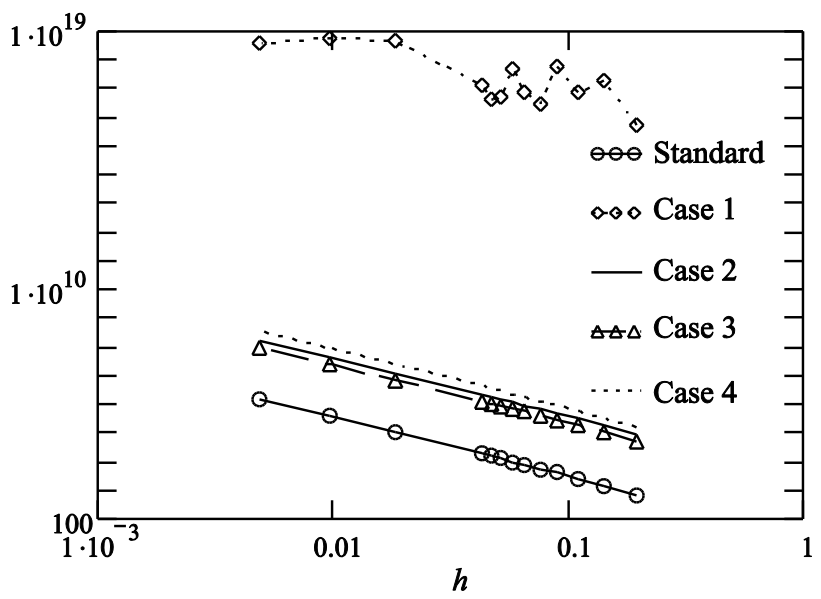

Fig. 8. Condition number of the stiffness matrix according to $L_{2}$ norm 

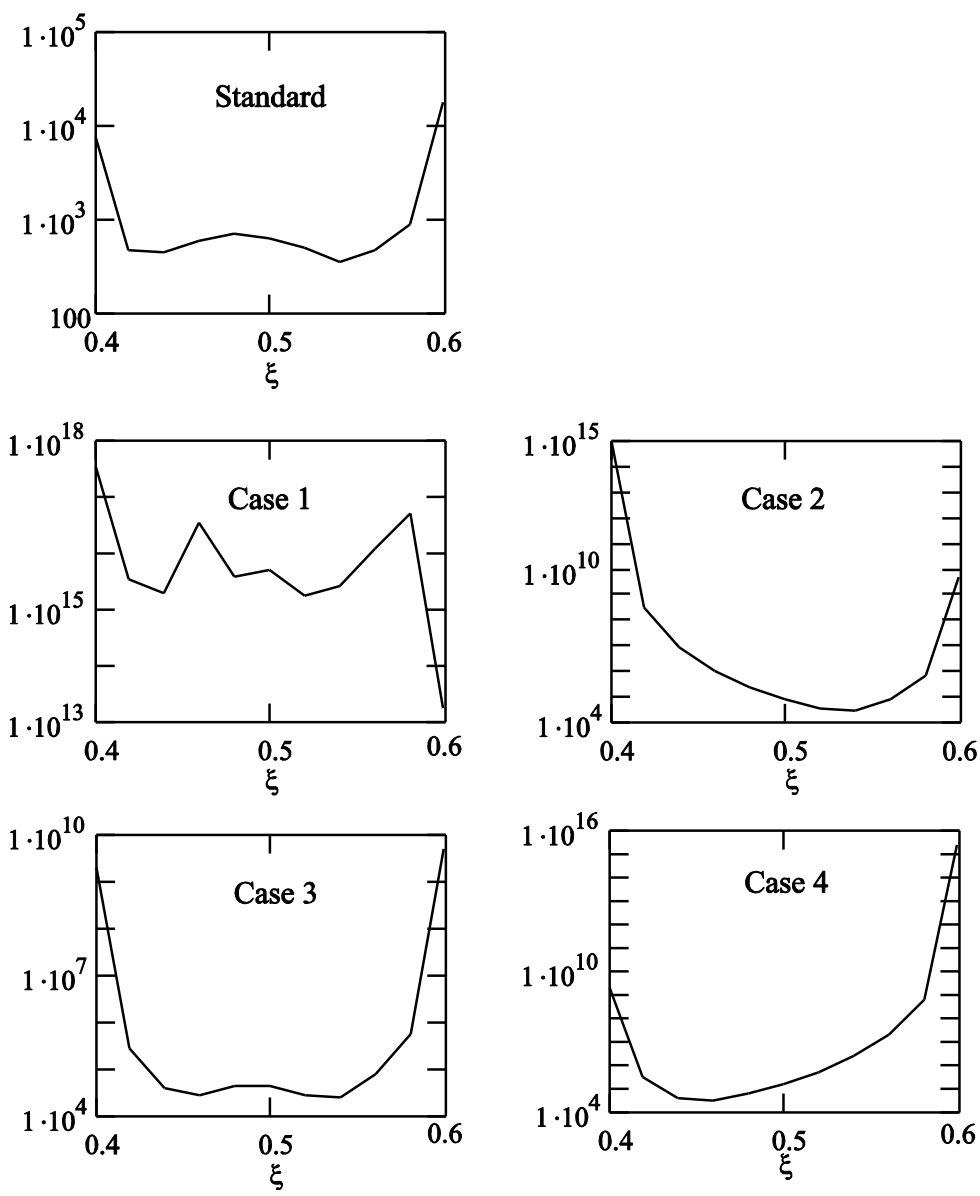

Fig. 9. Condition number in relation to the interface position $\xi$

of the interface on the condition number is shown in Fig. 9, where the log scales are used for vertical axes. As can be observed, the condition number increases considerably if the position of the interface approaches to the element's boundaries. This phenomena is well-known in the XFEM, and can be treated by removing enrichment for the nodes if they are too close to the interface [19].

\section{Extension to two-dimensional case}

The PDE of Poisson type represents the problem in two-dimensional space

$$
\frac{\partial}{\partial x}\left(k_{x}(x, y) \frac{\partial}{\partial x} u(x, y)\right)+\frac{\partial}{\partial y}\left(k_{y}(x, y) \frac{\partial}{\partial y} u(x, y)\right)+f(x, y)=0 .
$$

The Dirichlet and Neumann boundary conditions take the form

$$
u(x, y)=u_{D} \text { at } \Gamma_{D}
$$




$$
\left[k_{x}(x, y) \frac{\partial}{\partial x} u(x, y) \quad k_{y}(x, y) \frac{\partial}{\partial y} u(x, y)\right] \mathbf{n}=q_{N} \quad \text { at } \quad \Gamma_{N},
$$

where $\mathbf{n}$ denotes the normal vector to the boundary $\Gamma$, (Fig. 10).

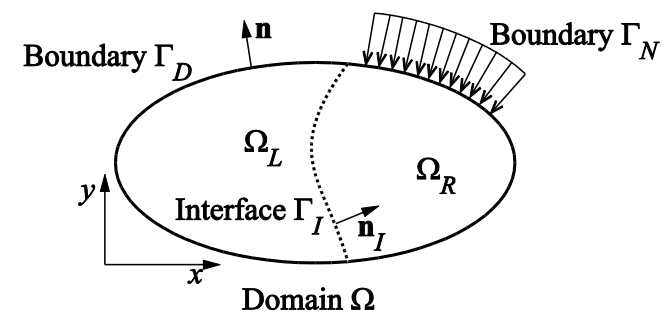

Fig. 10. Two-dimensional domain $\Omega$ split into $\Omega_{L}$ and $\Omega_{R}$ by the interface $\Gamma_{I}$

As for the one-dimensional case, it is assumed that the coefficients $k_{x}(x, y)$, $k_{y}(x, y)$ and function $f(x, y)$ are explicitly discontinuous across the interface boundary $\Gamma_{I}$

$$
\begin{aligned}
& k_{x}(x, y)=\left\{\begin{array}{lll}
k_{L}^{x}(x, y) & \text { in } & \Omega_{L} \\
k_{R}^{x}(x, y) & \text { in } & \Omega_{R}
\end{array}\right. \\
& k_{y}(x, y)=\left\{\begin{array}{lll}
k_{L}^{y}(x, y) & \text { in } & \Omega_{L} \\
k_{R}^{y}(x, y) & \text { in } & \Omega_{R}
\end{array}\right. \\
& f(x, y)=\left\{\begin{array}{lll}
f_{L}(x, y) & \text { in } & \Omega_{L} \\
f_{R}(x, y) & \text { in } & \Omega_{R} .
\end{array}\right.
\end{aligned}
$$

In the two-dimensional case the explicit description of discontinuity becomes cumbersome. The most popular approach to tackle this problem is implicit way of defining the position of discontinuity using level set method. In the level set method the position of the interface $\Gamma_{I}$ is defined as a zero-level set of a higher-dimensional surface $\phi$ such that

$$
\Gamma_{I}=\{\mathbf{x}: \phi(x, y)=0\}
$$

where vector $\mathbf{x}$ denotes the coordinates $(x, y)$ of a point.

The level set function $\phi$ is generally regarded as a signed distance function

$$
\phi(x, y)= \pm \min _{x_{I} \in \Gamma_{I}}\|\mathbf{x}-\xi\| \quad \forall \mathbf{x} \in \Omega
$$

where $\xi$ is the vector representing coordinates of a point at the interface $\Gamma_{I}$. The vectors normal to the interface front at any point of the domain can thus be calculated as follows

$$
\mathbf{n}_{I}(x, y)=\frac{\nabla \phi(x, y)}{\|\nabla \phi(x, y)\|}
$$


The level set function allows us to address in a simple way the most important XFEM issues, namely: defining the position of the interface, defining the enrichment functions directly, and deciding where the enrichment should be carried out.

As the nodal values of the level set function $\phi\left(\mathbf{x}_{i}\right)$ are known, the set of enriched nodes $J$ can be easily found according to

$$
J=\left\{k \in\{1, \ldots, e\}: \min _{i \in I_{k}^{e}}\left(\phi\left(\mathbf{x}_{i}\right)\right) \max _{i \in I_{k}^{e}}\left(\phi\left(\mathbf{x}_{i}\right)\right)<0\right\}
$$

where $I_{k}^{e}$ is the set of nodes belonging to the element $e$.

The approximation formula (9) written in the matrix form for two-dimensional case is

$$
u_{h}(\mathbf{x})=\mathbf{N}_{U}(\mathbf{x}, \boldsymbol{\xi}) \mathbf{u}_{X}
$$

where $\mathbf{N}_{U}$ and $\mathbf{u}_{X}$ are the extended shape function vector and the extended degrees of freedom vector, respectively; they are defined as

$$
\begin{aligned}
& \mathbf{N}_{U}(\mathbf{x}, \boldsymbol{\xi})=\left[\begin{array}{ll}
\mathbf{N}(x) & \mathbf{N}(\mathbf{x}) \boldsymbol{\Psi}_{j}(\mathbf{x}, \boldsymbol{\xi})
\end{array}\right] \\
& \mathbf{u}_{X}=\left[\begin{array}{c}
\mathbf{u} \\
\mathbf{a}_{j}
\end{array}\right]
\end{aligned}
$$

The extension of the XFEM approximation to the two-dimensional space requires definition of the enrichment function for the two-dimensional finite element. Four-node (rectangular) finite elements with bi-linear shape functions are used in discretisation. In order to allow accurate integration of discontinuous functions in two-dimensions, an a-split scheme is adopted according to Delaunay triangulation.

In the study, the standard abs-enrichment with shifted-basis approximation

$$
\Psi_{j}(\mathbf{x})=|\phi(\mathbf{x})|-\left|\phi\left(\mathbf{x}_{j}\right)\right|
$$

is compared to the enrichment functions proposed by Moës et al. [18]

$$
\Psi^{M}(\mathbf{x})=\mathbf{N}(\mathbf{x})|\phi|-|\mathbf{N}(\mathbf{x}) \phi|
$$

and the new set of enrichment functions proposed by authors

$$
\begin{gathered}
\Psi^{1}(\mathbf{x})=\Psi^{M}(\mathbf{x}) \\
\Psi_{j}^{2}(\mathbf{x})=\Psi^{M}(\mathbf{x}) \frac{1+H(\mathbf{x}) H\left(\mathbf{x}_{j}\right)}{2 \mathbf{N}(\mathbf{x})|\phi|}+\frac{1-H(\mathbf{x}) H\left(\mathbf{x}_{j}\right)}{2}
\end{gathered}
$$

where

$$
H(\mathbf{x})= \begin{cases}-1 & \text { if } \quad \phi(\mathbf{x})<0 \\ +1 & \text { if } \phi(\mathbf{x}) \geqslant 0\end{cases}
$$


The proposed enrichment functions (47) and (48) combine the arbitrary function with the constant terms. The graphical representation of the functions (48) for an four node finite element and an arbitrary discontinuity is presented in Fig. 11. The Heaviside function is used in order to eliminate a part of the approximation in one region. This allows one to construct the enrichment term using two kinds of functions, ensuring continuity of the function at the interface. They are incorporated to the final shape function in the same way as in the equation (26). For both (46) and (47)-(48) cases, the enrichment vanishes in blending elements so the optimal convergence rates are expected.
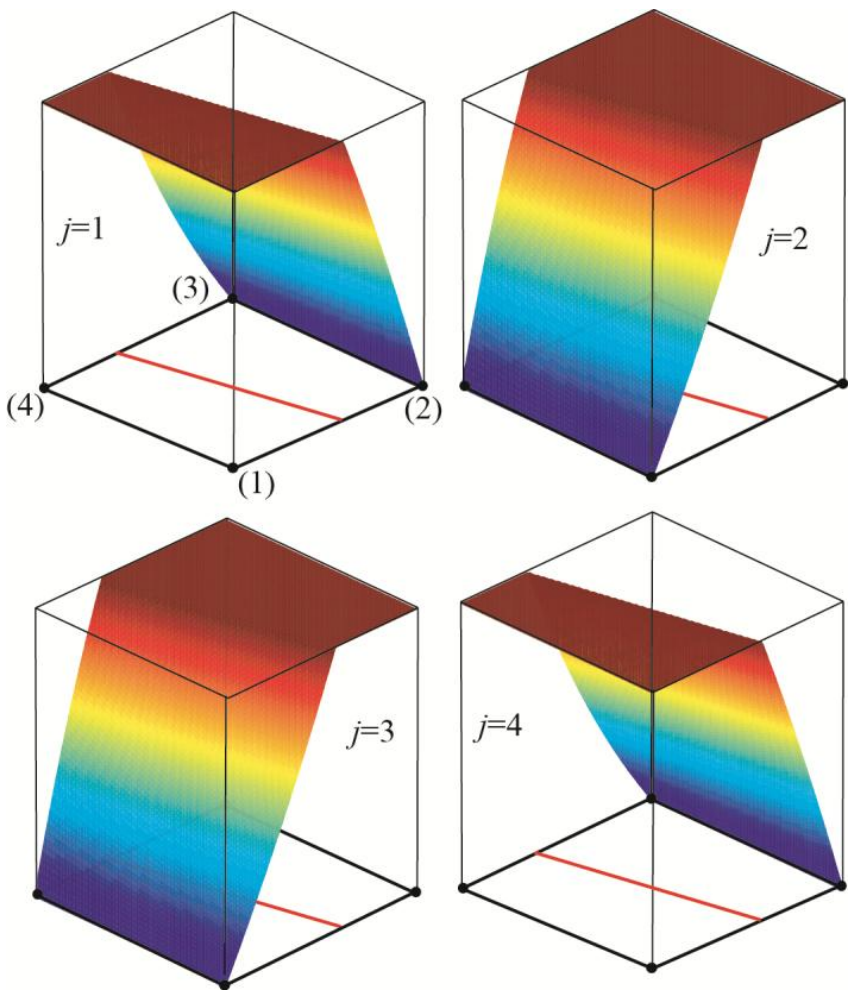

Fig. 11. The graphical representation of the enrichment functions $\Psi_{j}^{2}(\mathbf{x})$ for a four node finite element

\section{Two-dimensional numerical example with straight interface}

The example presents a basic two-dimensional discontinuous problem defined on the domain $(x, y) \in \Omega=[0,1]^{2}$ with a straight vertical interface [20]. The Dirichlet boundary conditions are prescribed at the boundaries

$$
\left.u\right|_{x=0}=\left.u\right|_{y=0}=\left.u\right|_{y=1}=0 \quad \text { and }\left.\quad u\right|_{x=1}=\sin (\pi y)
$$


The level set function becomes zero at the vertical line $x=2 / 3$

$$
\phi(x, y)=-x+\frac{2}{3} .
$$

We choose the forcing term $f=0$ and the coefficients such that

$$
\begin{aligned}
& k_{x}=\left\{\begin{array}{lll}
1 & \text { if } \phi(\mathbf{x})<0 \\
10 & \text { if } \phi(\mathbf{x}) \geqslant 0
\end{array}\right. \\
& k_{y}=\left\{\begin{array}{lll}
1 & \text { if } \phi(\mathbf{x})<0 \\
1 & \text { if } \quad \phi(\mathbf{x}) \geqslant 0 .
\end{array}\right.
\end{aligned}
$$

The analytical solution is then [20]

$$
u(x, y)= \begin{cases}2 A \sin (\pi y) \sinh (\alpha x) & \text { if } \phi(\mathbf{x})<0 \\ B \sin (\pi y)\left[\left(e^{\beta x}-e^{\beta(2-x)}\right)+e^{\beta(1-x)}\right] & \text { if } \phi(\mathbf{x}) \geqslant 0\end{cases}
$$

where $\alpha=\pi, \beta=-\sqrt{\frac{\pi^{2}}{10}}, A=0.1074$ and $B=0.5625$. The solution is based on the calculated numbers $A$ and $B$, thus the formula (54) is treated as a reference solution rather than the exact solution.

The numerical XFEM solution obtained for $8 \times 8$ element mesh is presented in Fig. 12. Four-node, bi-linear finite elements are used in the discretisation. The error distribution calculated as the difference of the analytical and numerical solutions is shown in Fig. 13, 14 and 15 for three enrichments considered, namely: standard abs-enrichment (45), Moës et al. enrichment (46) and the proposed enrichment

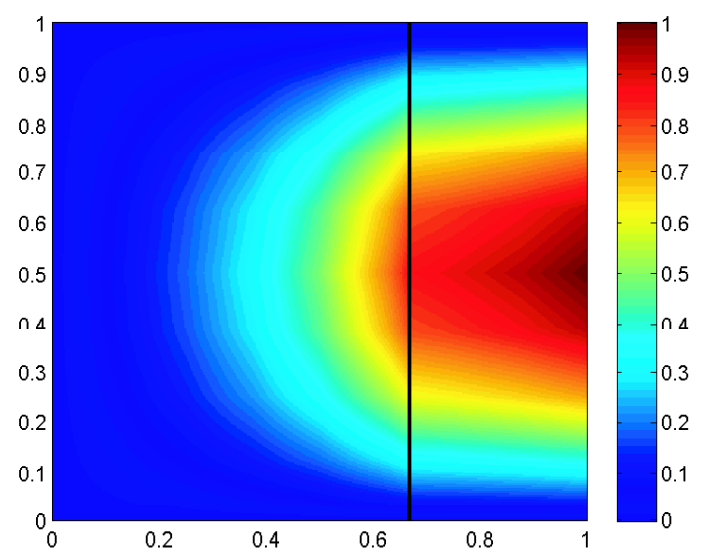

Fig. 12. The XFEM solution for a weak vertical discontinuity problem $(8 \times 8$ quadrilateral element mesh) 


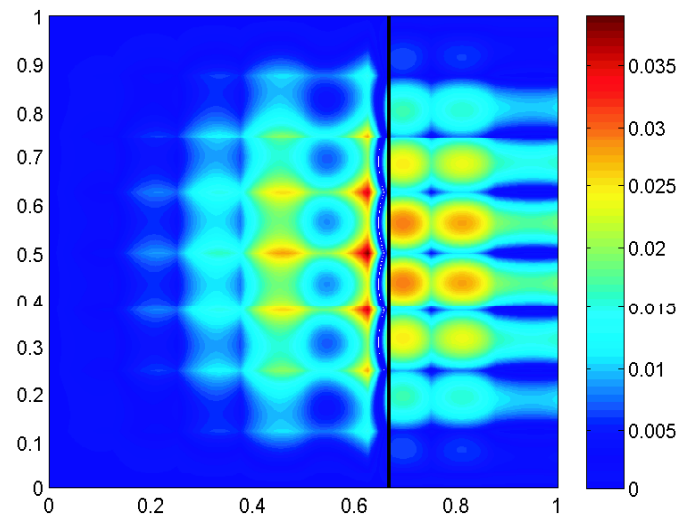

Fig. 13. Error distribution for a weak vertical discontinuity problem $(8 \times 8$ quadrilateral element mesh) and standard abs-enrichment

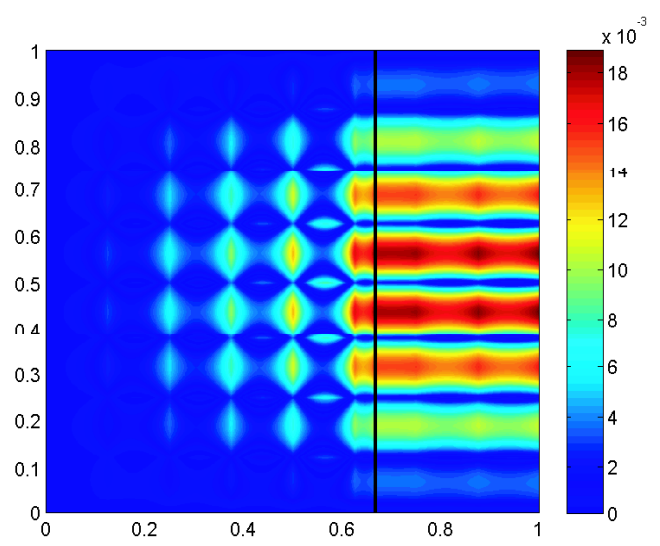

Fig. 14. Error distribution for a weak vertical discontinuity problem $(8 \times 8$ quadrilateral element mesh) and special enrichment proposed by Moës et al. [18]

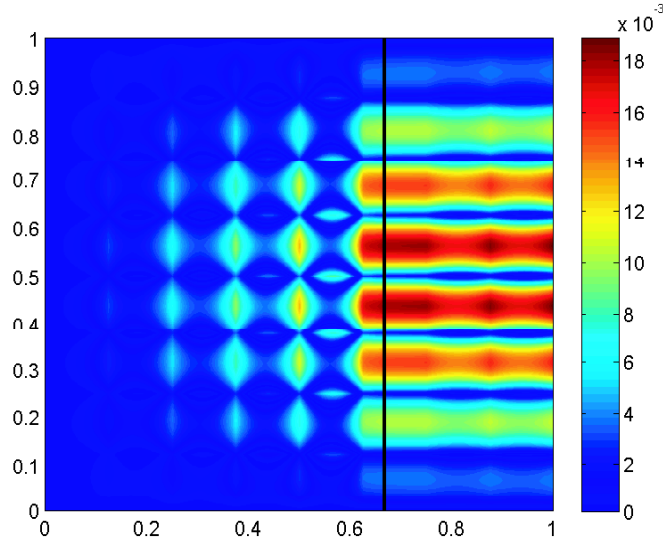

Fig. 15. Error distribution for a weak vertical discontinuity problem $(8 \times 8$ quadrilateral element mesh) and enrichment proposed 
(47)-(48). The rates of convergence of the norm for the obtained solutions and condition numbers of the stiffness matrix are shown in Fig. 16 and 17, respectively. The $L^{2}$ norm is defined as the size of the error between the exact and XFEM solutions

$$
L^{2}=\left\|u(x)-u_{X F E M}(x)\right\| .
$$

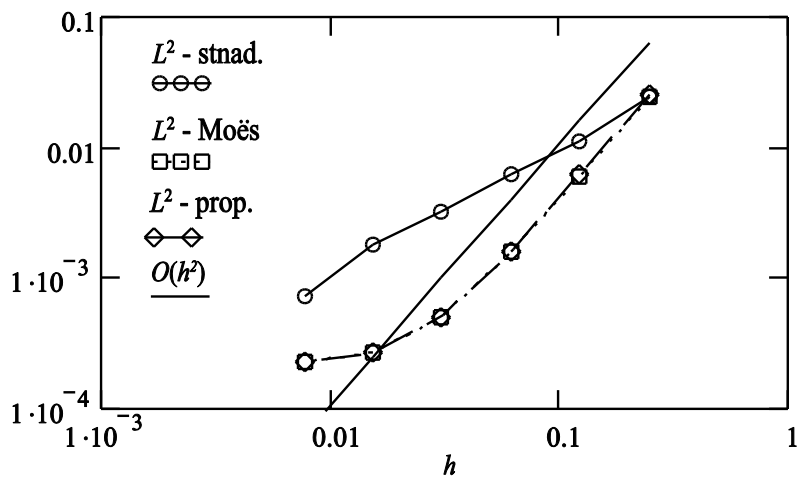

Fig. 16. Convergence results for $L^{2}$ norm (abbreviations: stand. - standard abs-enrichment, prop. proposed by author)

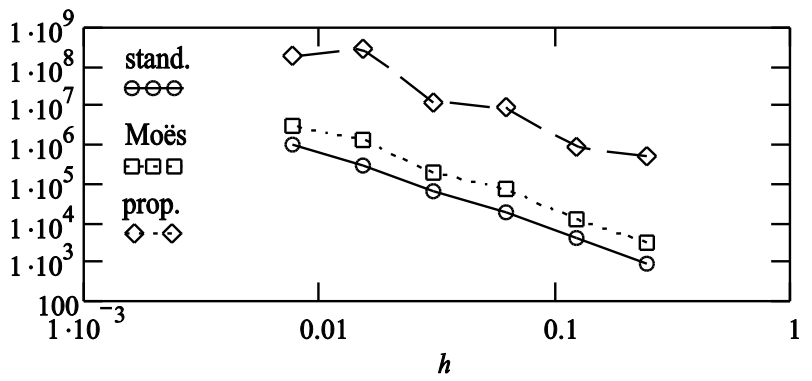

Fig. 17. Condition number of the stiffness matrix

As can be observed, the Moës and proposed enrichment functions allows for reducing the error in $L_{2}$ norm and improving the rate of convergence. The same reduction in convergence rate is also observed for triangular elements.

Fig. 18, 19 and 20 compare the exact derivative $\frac{\partial}{\partial x} u(x, y=0.5)$ with computed derivative using $4 \times 4$ element mesh and three considered approximations. The rough mesh used in the calculation shows an ability of the considered approaches to mimic local behaviour of the solution.

The local error (32) calculated along the interface line is shown in Fig. 21 and 22. The proposed enrichment functions allows for reducing the error considerably for each considered mesh. The maximum values are observed in the middle point of the interface line. As in the one - dimensional case the improvement is obtained at the cost of higher condition number of the stiffness matrix. 


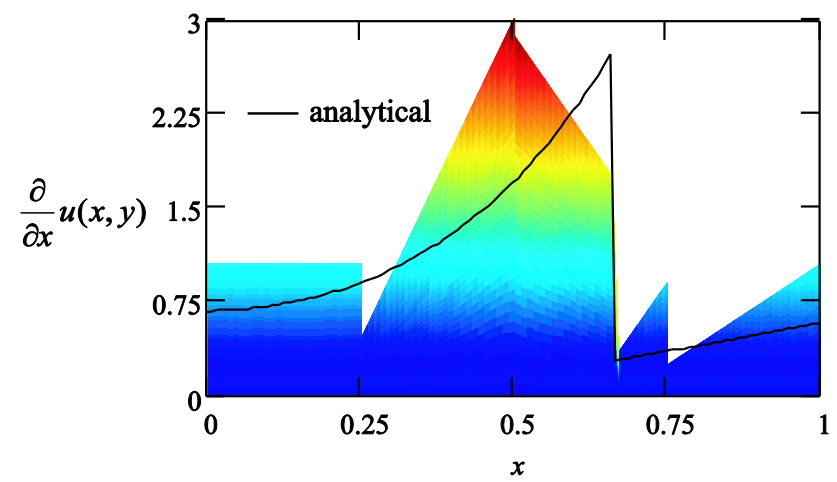

Fig. 18. Derivative $\frac{\partial}{\partial x} u(x, y=0.5)$ for the $4 \times 4$ element mesh and standard abs-enrichment

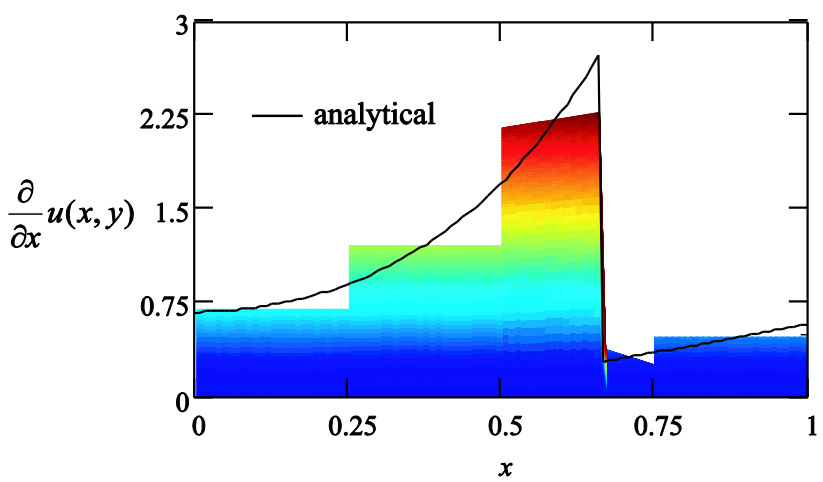

Fig. 19. Derivative $\frac{\partial}{\partial x} u(x, y=0.5)$ for the $4 \times 4$ element mesh and enrichment proposed by Moës et al. [18]

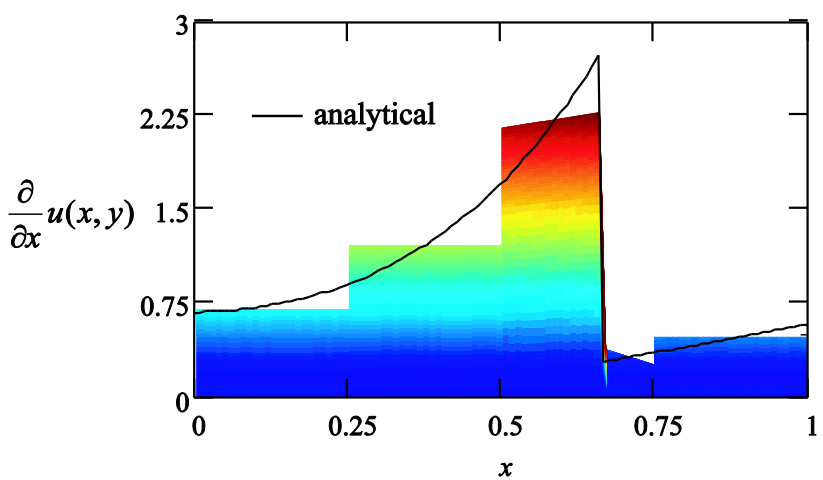

Fig. 20. Derivative $\frac{\partial}{\partial x} u(x, y=0.5)$ for the $4 \times 4$ element mesh and proposed enrichment 


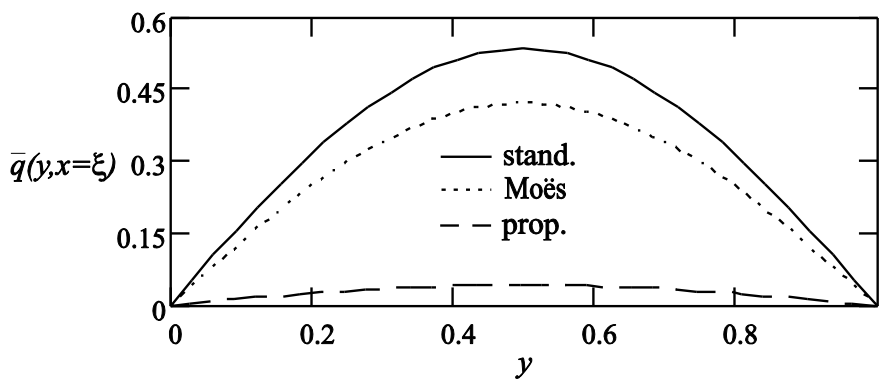

Fig. 21. Local error estimate $\bar{q}$ along the interface line for the $16 \times 16$ element mesh

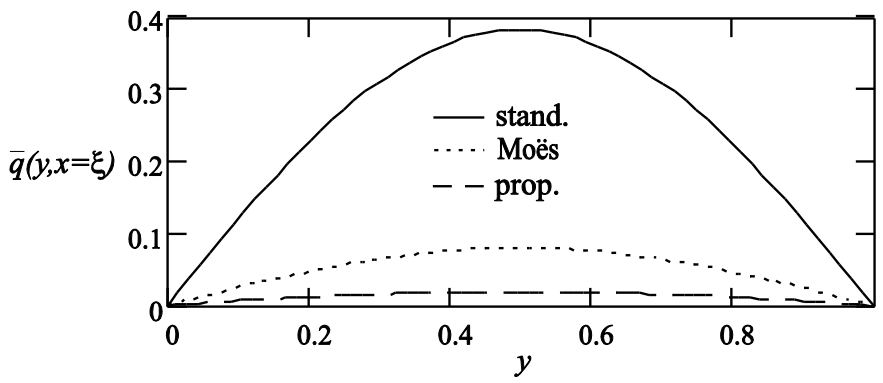

Fig. 22. Local error estimate $\bar{q}$ along the interface line for the $32 \times 32$ element mesh

\section{Two-dimensional numerical example with multiple, curved interfaces}

In the previous section, the method was applied to the problem with a straight vertical discontinuity, where the interface could be exactly represented by the FE representation of the level set function. With curved discontinuity this is no longer the case. In this section, we consider a problem with a curved and circular interfaces. The multiple interfaces can be easily handled with the level set function. In order to describe the interface between the domains, the continuous level set function is introduced

$$
\phi(x, y)=\min \left(\phi_{1}(x, y), \phi_{2}(x, y)\right)
$$

where

$$
\phi_{1}(x, y)=\sqrt{(x=0.25)^{2}+(y-0.25)^{2}}-0.15
$$

represents the circular inclusion and

$$
\phi_{2}(x, y)=\frac{\sqrt{2}}{2}-\frac{\sqrt{2}}{8} y^{2}-x
$$

represent the curved open interface. The FE representation of the zero-level for the function (56) is shown in Fig. 23 for the $20 \times 20$ element mesh. 


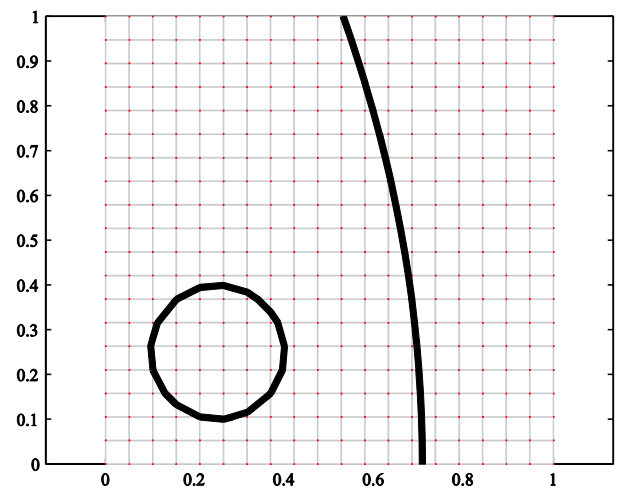

Fig. 23. FE representation of the level set function for the $20 \times 20$ element mesh

It is assumed that the source term is non-zero for the negative value of the level set function

$$
f(x, y)= \begin{cases}100 & \text { if } \phi(\mathbf{x})<0 \\ 0 & \text { if } \phi(\mathbf{x}) \geqslant 0\end{cases}
$$

and the parameters represent isotropic material

$$
k_{x}=k_{y}= \begin{cases}15 & \text { if } \phi(\mathbf{x})<0 \\ 1 & \text { if } \phi(\mathbf{x}) \geqslant 0\end{cases}
$$

The boundary conditions remains unchanged. The steady-state solution is presented in Fig. 24. For this example, the condition number increase is investigated, Fig. 25. As for the previous example, a similar relation is observed. The problem shows the possibility of the level set approach to construct the arbitrary multiple interfaces.

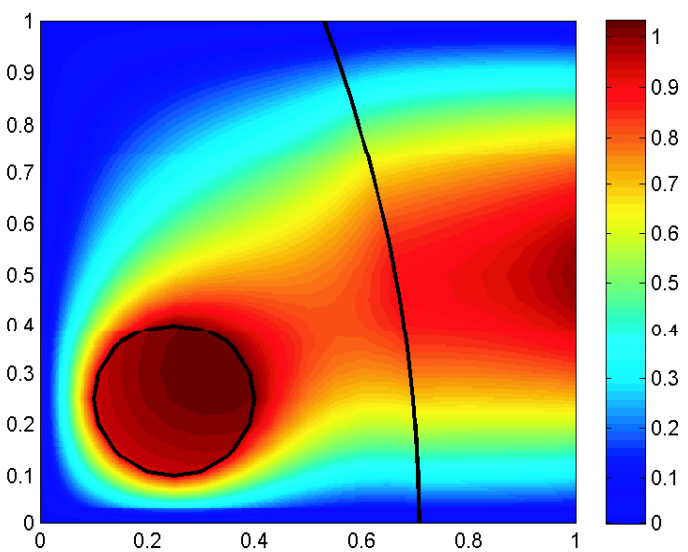

Fig. 24. Steady-state XFEM solution of the problem with a circular and curved interface for the $20 \times 20$ element mesh 


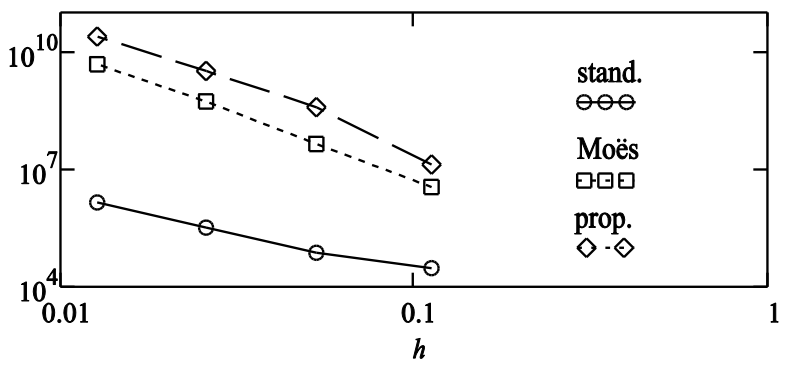

Fig. 25. Condition number of the stiffness matrix for the problem with multiple interfaces

\section{Conclusions}

In the paper, the solution to a boundary problem with discontinuous coefficients using XFEM is considered. The method is tested on the example of the Poisson equation in one and two-dimensional space. The standard XFEM approximation is extended to the new set of functions. The proposed improvements reduce considerably the analysis error in comparison to the standard approach. The rates of convergence for all considered cases of approximation are not disturbed as for both the and energy norms. However, the improvement in the calculated field quantity and its derivative is achieved at the cost of higher condition number of the underlying stiffness matrix.

Manuscript received by Editorial Board, June 04, 2016; final version, January 16, 2017.

\section{References}

[1] T.P. Fries and H.G. Matthies. Classification and overview of meshfree methods. Informatikbericht Nr.: 2003-3. Technical University Braunschweig, Brunswick, Germany, 2004.

[2] M.A. Schweitzer. Meshfree and generalized finite element methods. Postdoctoral dissertation. Mathematisch-Naturwissenschaftlichen Fakultat der Rheinischen Friedrich- -Wilhelms-Universitat, Bonn, Germany, 2008.

[3] Vinh Phu Nguyen, C. Anitescu, S. Bordas, and T. Rabczuk. Isogeometric analysis: An overview and computer implementation aspects. Mathematics and Computers in Simulation, 117:89-116, 2015. doi: 10.1016/j.matcom.2015.05.008.

[4] T. Belytschko and T. Black. Elastic crack growth in finite elements with minimal remeshing. International journal for numerical methods in engineering, 45(5):601-620, 1999.

[5] R. Merle and J. Dolbow. Solving thermal and phase change problems with the eXtended finite element method. Computational mechanics, 28(5):339-350, 2002. doi: 10.1007/s00466-0020298-y.

[6] J. Chessa, P. Smolinski, and T. Belytschko. The extended finite element method (XFEM) for solidification problems. International Journal for Numerical Methods in Engineering, 53(8):1959-1977, 2002. doi: 10.1002/nme.386.

[7] P. Stapór. The XFEM for nonlinear thermal and phase change problems. International Journal of Numerical Methods for Heat \& Fluid Flow, 25(2):400-421, 2015. doi: 10.1108/HFF-022014-0052. 
[8] J.Y. Wu and F.B. Li. An improved stable XFEM (Is-XFEM) with a novel enrichment function for the computational modeling of cohesive cracks. Computer Methods in Applied Mechanics and Engineering, 295:77-107, 2015. doi: 10.1016/j.cma.2015.06.018.

[9] P. Hansbo, M.G. Larson, and S. Zahedi. A cut finite element method for a stokes interface problem. Applied Numerical Mathematics, 85:90-114, 2014. doi: 10.1016/j.apnum.2014.06.009.

[10] E. Wadbro, S. Zahedi, G. Kreiss, and M. Berggren. A uniformly well-conditioned, unfitted nitsche method for interface problems. BIT Numerical Mathematics, 53(3):791-820, 2013. doi: 10.1007/s10543-012-0417-x.

[11] I. Babuška and U. Banerjee. Stable generalized finite element method (SGFEM). Computer Methods in Applied Mechanics and Engineering, 201:91-111, 2012. doi: 10.1016/j.cma.2011.09.012.

[12] K. Kergrene, I. Babuška, and U. Banerjee. Stable generalized finite element method and associated iterative schemes; application to interface problems. Computer Methods in Applied Mechanics and Engineering, 305:1-36, 2016. doi: 10.1016/j.cma.2016.02.030.

[13] G. Zi and T. Belytschko. New crack-tip elements for xfem and applications to cohesive cracks. International Journal for Numerical Methods in Engineering, 57(15):2221-2240, 2003. doi: 10.1002/nme.849.

[14] G. Ventura, E. Budyn, and T. Belytschko. Vector level sets for description of propagating cracks in finite elements. International Journal for Numerical Methods in Engineering, 58(10):15711592, 2003. doi: 10.1002/nme.829.

[15] J.E. Tarancón, A. Vercher, E. Giner, and F.J. Fuenmayor. Enhanced blending elements for XFEM applied to linear elastic fracture mechanics. International Journal for Numerical Methods in Engineering, 77(1):126-148, 2009. doi: 10.1002/nme.2402.

[16] T.P. Fries. A corrected XFEM approximation without problems in blending elements. International Journal for Numerical Methods in Engineering, 75(5):503-532, 2008. doi: 10.1002/nme.2259.

[17] P. Stąpór. Application of xfem with shifted-basis approximation to computation of stress intensity factors. Archive of Mechanical Engineering, 58(4):447-483, 2011. doi: 10.2478/v10180011-0028-0.

[18] N. Moës, M. Cloirec, P. Cartraud, and J.-F. Remacle. A computational approach to handle complex microstructure geometries. Computer methods in applied mechanics and engineering, 192(28):3163-3177, 2003. doi: 10.1016/S0045-7825(03)00346-3.

[19] J. Dolbow, N. Moës, and T. Belytschko. Discontinuous enrichment in finite elements with a partition of unity method. Finite elements in analysis and design, 36(3):235-260, 2000. doi: 10.1016/S0168-874X(00)00035-4.

[20] B.A. Saxby. High-order XFEM with applications to two-phase flows. PhD thesis, The University of Manchester, Manchester, UK, 2014. www.escholar.manchester.ac.uk/uk-ac-manscw:234445. 